\title{
Chapter 2 \\ eIoT Activates the Grid Periphery
}

Perhaps nowhere will the impact of the energy-management change drivers identified in Chap. 1 be felt more than at the grid's periphery. DG in the form of solar PV and small-scale wind will be joined by a plethora of internet-enabled appliances and devices to transform the grid's periphery to one with two-way flows of power and information $[45,46]$. This transformation presents a daunting technical challenge. Not only are there tens of millions of devices at the leaves of the grid's radial structure, these devices are relatively small and require new innovations in sensing, communication, control, and actuation.

This chapter first describes this transformation in Sect. 2.1. Section 2.2 describes the challenge of activating the grid's periphery. Finally, Sect. 2.3 describes how eIoT can potentially be deployed as a scalable energy-management solution.

\subsection{Change Drivers Will Transform Energy Management at the Grid Periphery}

The installation of DG in the form of solar PV and small-scale wind causes twoway flows of power and information at the grid periphery. The change drivers discussed in Sect. 1 directly and indirectly incentivize growth in renewable energy generation. Renewable energy is, by nature, decentralized, and the deployment of small-scale power generation is increasing in industrial, commercial, and residential applications [134]. For example, the installations of solar PV systems in the USA nearly doubled from 2014 to 2016 [134]. Generation at the grid periphery introduces

a power flow inward, or upward, towards the transmission system in addition to the normal outward power flow to consumers.

As the generation at the periphery of the grid continues to grow, energymanagement systems must adjust from a "top-down" hierarchical structure of communication and control to one that is more dynamic and distributed [135]. The variable nature of renewable energy resources (for example, solar PV and wind)

S. O. Muhanji et al., eIoT, https://doi.org/10.1007/978-3-030-10427-6_2 


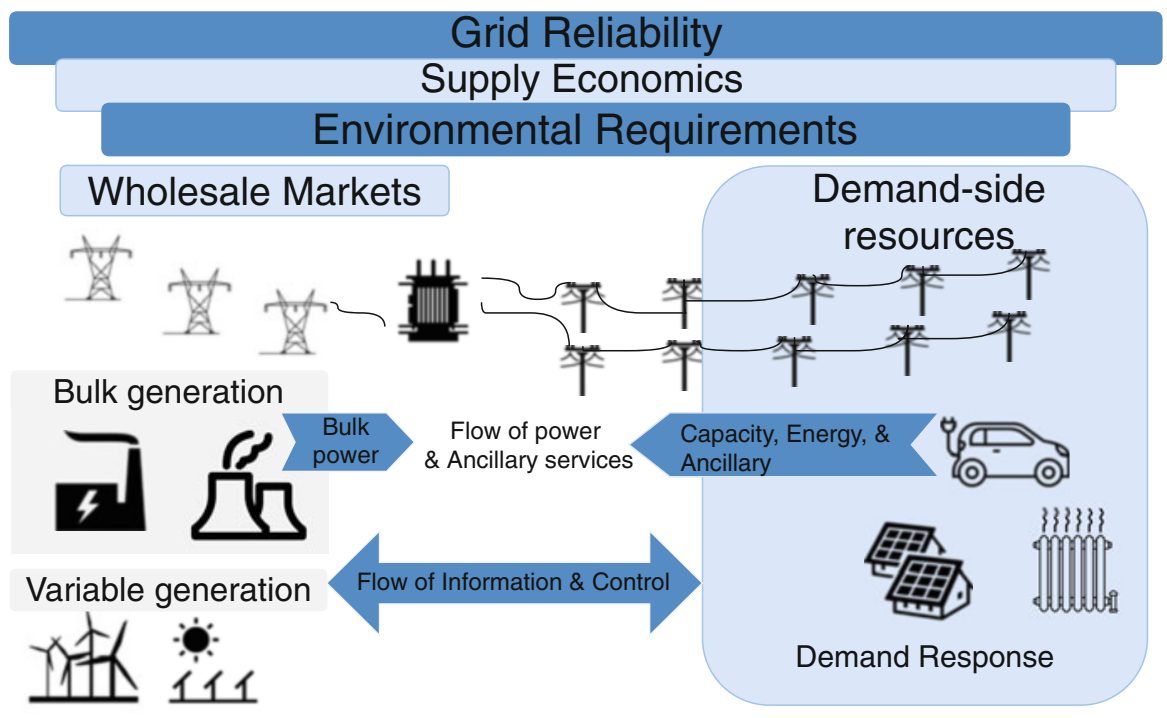

Fig. 2.1 A grid periphery activated by variable generation and demand response (adapted from [14])

means that in order to achieve sustainability, data acquisition, and new networks to monitor real-time power flows are imperative [136]. This is best illustrated in Fig. 2.1 which shows the need for two-way flow of information and control between the grid generation and transmission system and the grid periphery with a large penetration of distributed generation and demand response.

In addition to DG, a plethora of internet-enabled appliances and devices further reinforce the presence of two-way flows of power and information at the grid periphery. The demand side provides devices for controlling the balance of power consumption and generation through real-time demand response. High penetration rates of renewable energy motivate the need for real-time demand response; furthermore, deregulation and increased consumer participation is achieved with active economic real-time demand response. IoT devices, at the periphery, such as electrified vehicles (EVs); electricity storage in industry, commercial buildings, and residences; and smart devices in the home have created a new demand-side network of devices that requires the grid to become more dynamic as device interactions increase [97].

With drivers to incorporate DER and DR programs, smart grid technologies will enable end users to actively manage their electric loads according to price incentives. This active balancing of power at the grid periphery can shift in real time from positive (due to excess DG) to negative (due to modulated/controlled/incentivized) demand response. Internet-enabled appliances and devices in the grid periphery must be monitored and controlled in order to take advantage of real-time shifts in economic demand response. Bidirectional information flow sends pricing signals to the devices, while device information is sent to the controller. Where feedback 
loops are physical rather than economic, these devices can also potentially provide ancillary services in response to operational signals, for example, grid frequency, voltage, and line congestion.

The need to monitor and control two-way flows of power with two-way flows of information emphasizes the role of data gathering in the power grid. Data are needed to make accurate control decisions in the grid's increasingly flexible and fast-paced environment. Utilities are deploying more devices to collect more data of increasing diversity. The global number of devices being managed by utility companies is projected to grow from 485 million in 2013 to approximately 1.53 billion in 2020 [97]. Improved grid monitoring and control involves increasing the quantity of field distribution automation devices, field monitoring devices, substation monitoring and control, and interconnections and monitoring of independent power producers (IPPs) [137].

Also, future utility investments are expected to develop smart metering infrastructure across industrial, commercial, residential, transformer, and field meters [137]. Each application should accommodate a utility's business model and the network's specifications. For example, field distribution automation devices include remote monitoring and control of distribution reclosers, switches, voltage regulators, and capacitor banks that must be united under a common communication network [137]. All of these devices produce data at regular intervals, although there is a shift towards real-time data streaming. For instance, some smart sensor systems produce large streams of data from thousands of sensors, which-without appropriate planning and design-have the potential to overload system operators [138]. Due to the growing magnitude of deployed devices, and the use of proprietary and non-proprietary solutions, the monitoring devices on the grid produce increasingly heterogeneous data [139]. More devices, recording ever-more diverse measurements, create a thorough monitoring environment that has the potential to improve power system operations with new self-healing and reconfiguration capabilities. Granular data will also shift the grid from load-following to loadshaping energy management [3].

In order to support the two-way flows of information in the power grid, new networks are necessary. Many smart devices use applications that depend on data sets distributed across many devices. Furthermore, this information is often relayed to centralized centers for further storage, processing, and decision-making [140]. Multiple types of networks are required to co-exist. Although the supervisory control and data acquisition (SCADA) system gives utilities limited control of their upstream functions, the distribution network is insufficiently monitored and controlled [141].

As a solution, distribution-management solutions are expected to integrate with upstream SCADA as well as interoperate with the complex multitude of downstream network-enabled devices. In a survey sent to over 300 members of the Institute of Electrical and Electronics Engineers (IEEE) power energy system (PES) distribution-management system (DMS) task force, which comprises $76 \%$ utilities, about $72 \%$ of responders noted that SCADA facilities would be an integral part in distribution-management systems (DMS) [142]. Over $80 \%$ of survey participants 
also responded that more than one mechanism is necessary to handle DMS data acquisition and control requirements [142]. This is because SCADA's centralized and hierarchical structure is ill-suited for the developments in information and communication technology at the grid's periphery.

Because SCADA is a utility-purchased software that monitors hardware in the electricity infrastructure [143], consumer-owned smart devices are out of the realm of SCADA control. Therefore, consumer devices require either their own local area network (LAN) or access to a common network such as the internet. For example, a private solution-specified network may include machine-to-machine (M2M) systems that remotely read customer energy consumption and interface with power grid communications [97]. The IoT can further enhance the operational capabilities of M2M systems by connecting several such systems together [97]. Naturally, interoperability of the emerging networks is crucial. However, open network access raises privacy and security concerns. Cyber-security efforts must be directed towards individual devices as well as the communication channels between them. With many networks existing beyond the scope of the utility, these efforts are ever-more integral to the physical security of the grid.

As two-way flows of power and information become common place at the grid periphery, new energy market structures can evolve from their current hierarchy. The integration of renewable energy into market operations requires new measurements, measurement devices, and market information to ensure efficient and equitable operation [101]. As renewable energy and active demand-side resources become more prevalent, the grid's periphery will become not just a source of power, but also a place for diversified market activities [97]. As new market agents appear, they will require real-time measurements for market surveillance and contract compliance [101]. More specifically, DER incentives rely on bidirectional price and consumption data to be effective [144].

Grid and meter data can support the efficacy of these market mechanisms at both the wholesale and local levels. Furthermore, such data can help shape the development of monetized efficiency services based upon the real-time behaviors of residential, industrial, and commercial customers [97]. These trends, taken in the context of deregulation, encourage the participation of non-traditional parties [97]. DG, in particular, has the potential for large-scale market disruption. It is uncertain how the structure of energy markets will change as energy consumers evolve into prosumers [145].

\subsection{The Challenge of Activating the Grid Periphery}

The transformation of the grid periphery is a daunting technical challenge because it is characterized by millions of small devices; all of which need to be coordinated to achieve high-level technical and economic energy-management objectives. For example, actively shaping the load profile when it is composed of so many devices 
is a great challenge as it requires precision control, accurate forecasts, and flexible resources. Such a grid transformation poses integration challenges in operations as well as in the fiscal and strategic planning of distributed resources. Sensing equipment must improve to support demand-side management, and system planning requires cheaper devices that can be deployed at scale. In addition to extending sensing and control capabilities in the distribution system, other challenges in periphery management include inflexible loads.

The technical challenges of integrating the grid with peripheral devices in DR solutions, all through a consistent regulatory and economic framework, are staggering. The ongoing interconnection of the electric power system requires foresight and planning on the part of operators as well as regulators. All the while, the grid needs to be in full operation at its usual level of reliability and security.

Not only will the transformation of the grid periphery be complicated by their large number but also by their tremendous heterogeneity. This means that coordination and control algorithms must account for a wide variety of devices each with their own device-specific behaviors. "The future electric system will include a large network of devices that are not only passive loads, as most endpoints today are, but devices that can generate, sense, communicate, compute, and respond. In this context, intelligence will be embedded everywhere, from EVs and smart appliances to inverters and storage devices, from homes to microgrids to substations" [146].

Independent actors at the grid periphery are expected to add tens of millions of devices with different sizes, consumption patterns, time scales, and with different control and economic capabilities [146]. Such DERs (devices) include both generation and consumption. On the generation side, generation can be derived from wind energy systems, photovoltaic cells, microturbines, fuel cells, solar dishes, gas turbines, diesel engines, and gas-fired internal combustion engines [147]. Demandside resources would include smart appliances, EVs, water heaters, air-conditioners, and energy storage in homes, buildings, and factories [148]. DERs also make use of power electronic interfaces so as to connect flexibly to the grid [147]. The centralized control of such devices is limited to hundreds or even a few thousand monitoring and control points.

As such, the distribution system is ill-equipped to control and coordinate the millions of homes, buildings, and factories with their associated energy devices [148]. Each customer and device has the potential to independently and dynamically interact with grid operations and markets. Such cases would require the implementation of complex algorithms for monitoring and control [146].

Due to the small size of devices and their increasingly complex interactions, the distribution system needs to be controlled with even more precision. Power system performance, control and daily operation use various mathematical models that need accurate generation, transmission, and distribution parameters in order to run [149]. It is very difficult to control and coordinate a large number of devices so that they achieve positive global objectives, especially when distribution monitoring is inadequate. 
Such a multi-objective system coordination problem, that is, factoring not only improved system quality, security, customer service, and economics, requires more effective and robust control strategies [149]. Evaluating these different control options opens the question of whether the control architecture should exhibit hierarchy, heterarchy, or aspects of both. In the hierarchical system, linked aggregation points feed to a centralized control station. Aggregation is expected to be used in short-ranged sensor networks and connecting M2M networks with other technologies [150]. However, a comprehensive aggregation strategy is not clear. In heterarchy, control is distributed among centers with separated functions.

Present-day control centers are progressively characterized by separated control systems, energy-management models, data models, and middleware-based distributed energy-management system (EMS) and distribution-management system (DMS) applications [101]. Distribution control algorithms allow for scalability at pace with the growth of consumer nodes, but many suitable algorithms have yet to be developed. Most likely, the grid requires a mixture of aggregation and distribution philosophies to meet its diverse objectives.

To further complicate matters, the distribution system and grid periphery, unlike the transmission system, have not been traditionally monitored or controlled. Traditional, centralized control depends on independent system operator (ISO) supervision with the participation of large generators and load-serving entities. ISOs, however, cannot view the system past substations [151]. Essentially blind, operators are concerned about renewable generation at the periphery [148, 151]. ISOs currently aggregate variable net load at the transmission substation, which results in uncertainty that must be counterbalanced by expensive and inefficient operations, such as larger transmission and reserve capacity acquisition by the ISO and power providers [151].

Consequently, the activation of the grid periphery to include full control loops of sensing, decision-making, and actuation requires significant technology development and implementation. DERs must be visible and controllable by grid operators and planners in order to secure reliability and enhance economic efficiency. Such integration needs a framework for transmission, distribution, and demandside resources that includes new analysis tools, visualization capabilities, and communications, and control methods [144]. Naturally, any effective strategy has to assume that there will be a migration from traditional passive devices to an everincreasing but gradual penetration of network-enabled devices.

As more DG and network-enabled devices are integrated into power grid operations, utilities and grid operators are less able to accurately predict the stochastic net load profile. Since the inception of the electric grid, consumers have dictated the quantity of power that has been sourced by controllable generation. The design of the electric system was built on this paradigm; it was not intended for substantial amounts of uncontrollable generation, such as variable renewable energy [152]. In today's grid, operators turn on generators to meet a prediction of aggregated consumer demand. However, renewable energy's dispatchability (ability to dispatch to accurately meet demand) remains largely uncontrollable, and its predictability can change due to weather conditions and site-specific conditions [152, 153]. 


\begin{tabular}{|c|c|c|c|}
\hline \multirow[t]{2}{*}{ Past: } & & Generation/Supply & Load/Demand \\
\hline & & $\begin{array}{l}\text { Thermal Units: } \\
\text { (Few, Well-Controlled, } \\
\text { Dispatchable Resources) }\end{array}$ & $\begin{array}{l}\text { Conventional Loads: } \\
\text { (Fairly Slow Moving, Highly } \\
\text { Predictable, Always Served) }\end{array}$ \\
\hline \multirow[t]{3}{*}{ uture: } & & Generation/Supply & Load/Demand \\
\hline & $\begin{array}{c}\text { Well-Controlled \& } \\
\text { Dispatchable }\end{array}$ & $\begin{array}{l}\text { Thermal Units: } \\
\text { (Potential Erosion of } \\
\text { Capacity Factor) }\end{array}$ & $\begin{array}{c}\text { Demand Side Management: } \\
\text { (Requires new control } \\
\& \text { market design) }\end{array}$ \\
\hline & $\begin{array}{l}\text { Stochastic/ } \\
\text { Forecasted }\end{array}$ & $\begin{array}{l}\text { Solar \& Wind Generation: } \\
\text { (Variability can cause } \\
\text { unmanaged grid imbalances) }\end{array}$ & $\begin{array}{l}\text { Conventional Loads: } \\
\text { (Continuing source of } \\
\text { variability \& uncertainty) }\end{array}$ \\
\hline
\end{tabular}

Fig. 2.2 A future smart grid with stochastic and controllable supply- and demand-side resources [15-17]

As a result, forecast errors are expected to increase. Prediction models need to be individually developed per site, since local characteristics influence renewable power generation [152]. Utilities may develop such prediction models for largescale renewable generation, but it is impractical to invent a separate model for each residential and small-scale distributed generator [152]. Referencing Fig. 2.2, the increasing penetration of variable energy is analogous to shifting from controllable loads to stochastic loads, but operator management of the system at large does not change as quickly. Forecast error is a long-standing operational challenge that will continue to grow as the penetration of renewable energy generation increases. In the immediate future, operation and control of demand-side resources must be precise in controlling set points of frequency, voltage, and line flows. Furthermore, these set points must be responsive to the errors propagated by inaccurate forecasting.

While the need for accurate forecasting in grid operations is ever-increasing, cost barriers remain to the implementation of advanced monitoring. Equipment expenses and other implementation objectives combat pressures for heavy monitoring in the grid. Conventional monitoring and diagnostic systems require expensive wiring and regular maintenance [154]. In contrast, wireless sensor networks (WSNs) have been pursued for their low cost, rapid deployment, and flexibility [154]. To deploy at scale, utilities maximize the per unit investment cost of sensing. For example, a fifty-dollar sensor on a 50-mW unit is far more valuable than the same cost sensor on a 50-kW unit. Such costs act as barrier to entry despite market deregulation [155].

Centralized generators often do not support investment in distribution monitoring systems, not just because of their costs, but also because they shift market power to DERs [155]. However, sensor technology developers are actively driving down the price of sensors for their widespread adoption. For example, the Auto-ID Center-the organization accredited with the term "Internet of Things" — set a goal 
of decreasing the cost of RFID tags from upwards of $\$ 0.50$ to as low as $\$ 0.05$ per tag [156]. Lower costs must come from new technologies and methods and cannot depend on simple economies of scale [156].

Finally, it is important to recognize that the control and coordination of demandside resources is fundamentally more complex than supply-side resources. Besides operational challenges, short-term and long-term consumer behaviors will need to be altered through DER management and incentivized DR programs [46]. The ultimate objective of DR is to alter demand so as to enhance grid reliability and economic efficiency [46]. Nevertheless, it is complicated by the inflexibility and time-varying economic utility of loads. While supply-side management exists solely to serve demand, demand-side management (DSM) primarily supports a nonelectrical activity, such as driving a motor or heating a building. Any behavioral shift (by DR programs) to support the reliable operation of the power grid is often at odds with the original intention of electricity consumption. Furthermore, it is important to recognize that a consumer's preference for electric consumption is time varying and "meddling" with service may lead to discomfort [46].

Fundamentally speaking, economic utility depends on the application of electric consumption. The value delivered by $1 \mathrm{~kW}$ of electricity for one purpose is not the same as the value delivered by another $\mathrm{kW}$ for another purpose, even if the kilowatt is consumed by the same customer! For instance, a manufacturing plant using $10 \mathrm{~kW}$ gets much more value when the electricity is consumed by a machine on the shop floor than by the back office. Uncertain economic utility and imperfect behavioral response make the control and coordination of demand-side resources particularly difficult.

\subsection{Deploying eIoT as a Scalable Energy Management Solution}

This work argues that the challenges of activating the grid periphery, described in the Sect. 2.2, may be addressed by deploying eIoT as a scalable energy-management solution. In essence, the energy-management challenges described in the previous section may be viewed as a control loop where dispatchable devices, whether they are traditional large-scale centralized generators or millions of small-scale internetenabled devices, must meet the three power system control objectives of balanced operation, line congestion management, and voltage control. These objectives can be achieved despite the presence of disturbances such as customer load or variable energy generation from solar PV and wind resources.

Fortunately, eIoT is fundamentally a control loop consisting of small-scale sensing technologies, wireless and wired communication technologies, distributed control algorithms, and remotely controlled actuators. And yet, despite eIoT having 
all of the components of a scalable energy-management control loop, the challenge is to continue to integrate more of these technologies in such a fashion that the control objectives are achieved well into the future. Chapter 3 details the development of eIoT technologies in terms of their role in a control loop.

Open Access This chapter is licensed under the terms of the Creative Commons Attribution 4.0 International License (http://creativecommons.org/licenses/by/4.0/), which permits use, sharing, adaptation, distribution and reproduction in any medium or format, as long as you give appropriate credit to the original author(s) and the source, provide a link to the Creative Commons licence and indicate if changes were made.

The images or other third party material in this chapter are included in the chapter's Creative Commons licence, unless indicated otherwise in a credit line to the material. If material is not included in the chapter's Creative Commons licence and your intended use is not permitted by statutory regulation or exceeds the permitted use, you will need to obtain permission directly from the copyright holder. 